\title{
Microscopic observation of multispecies biofilm of various structures on whey concentration membranes ${ }^{1}$
}

\author{
A. N. Hassan, ${ }^{2}$ S. Anand, and M. Avadhanula \\ Midwest Dairy Foods Research Center, Dairy Science Department, South Dakota State University, Brookings 57007
}

\begin{abstract}
The objective of this study was to evaluate biofilm formation on polyamide reverse osmosis ( $\mathrm{RO})$ whey concentration membranes. Biofilms were observed with scanning electron and fluorescence microscopy. For scanning electron microscopy, pieces of $6-, 12-$, and 14-mo-old membranes were allowed to air dry at room temperature $\left(22^{\circ} \mathrm{C}\right)$ for $24 \mathrm{~h}$ followed by sputter coating with a 5-nm layer of gold and microscopic observations. Scanning electron microscopy images revealed that the hydrophilic layer, used to prevent membrane plugging, was not evenly distributed on the surface. Although this hydrophilic layer seemed to prevent the attachment of proteins, it supported biofilm formation. Three different structures of multispecies biofilm were observed on the retentate side of the membrane: 1) a mono layer, 2) a 3-dimensional structure of a dense matrix of extracellular polymeric substances where different types of bacterial cells were embedded, and 3) cell aggregates. In some of the biofilms, a smooth layer (shell) covered cell aggregates. In the 6-mo-old membranes, part of the shell layer was broken off. Biofilms as observed on the RO membrane were described as having a hill-and-valley type of structure, with hills showing a mushroom-like appearance and valleys comprising dense matrices of extracellular polymers with embedded bacterial cells. Fluorescence microscopy showed live cells on the surface of the biofilm. It is concluded that both cells in the deep layers of biofilm and surface cells may resist cleaning and sanitation. The extent of biofilm formation and the presence of live cells on RO membranes after regular clean in place cycles indicate the need for a more effective cleaning regimen customized for dairy separation systems.
\end{abstract}

Received October 6, 2009

Accepted January 28, 2010

${ }^{1}$ Published with the approval of the director of the South Dakota Agricultural Experiment Station as Publication Number 3639 of the Journal Series. This research was supported in part by Midwest Dairy Foods Research Center, St. Paul, MN.

${ }^{2}$ Corresponding author: Ashraf.Hassan@sdstate.edu
Key words: whey, reverse osmosis, biofilm, microstructure

\section{INTRODUCTION}

Separation of dairy fluids using semipermeable membranes has been used for decades to clarify, concentrate, and fractionate a variety of dairy products, including cheese whey. In industrial practices, milk or whey membrane separation units may operate continuously for up to $24 \mathrm{~h}$. A decision to shut off and clean is usually made based on flux data. Membrane fouling is a major operational problem that leads to reduced membrane performance and premature replacement (Muthukumaran et al., 2005). In addition to proteins and minerals, bacteria are considered a major fouling agent (Ridgway et al., 1983; Tang et al., 2009).

Biofilms are viable and nonviable single or multispecies communities of microorganisms embedded in extracellular polymeric substances (EPS) attached to a substratum (Chmielewski and Frank, 2003). The EPS may contain polysaccharides, proteins, phospholipids, teichoic and nucleic acids, and other polymers (Chmielewski and Frank, 2003). Formation of biofilms on dairy separation and concentration membranes may affect not only their performance but also the quality and safety of the final product. Bacterial cells in biofilms are much more resistant to antimicrobial agents than they are in their planktonic state (Stewart and Costerton, 2001; Chmielewski and Frank, 2003). Therefore, biofilms may serve as a continuous contamination source for food-spoilage bacteria and pathogens in the finished product. Some recent studies on water treatment plants show that the decline in reverse osmosis (RO) membrane performance is caused by cell deposition and biofilm growth (Herzberg and Elimelech, 2007). There is limited information on the formation of biofilms on dairy separation membranes, especially whey RO systems. Recently, Tang et al. (2009) identified microbial species isolated from UF and RO whey processing membranes. They concluded that a variety of microorganisms, including pathogens, were associ- 
ated with dairy processing membranes, which indicated inadequate cleaning.

There is a need to develop novel, nondestructive methods for monitoring biofilm development. Some processors consider the membranes clean when permeate flux is restored after cleaning. However, flux is an indication of heavily contaminated membranes and cannot be considered a reliable measure of sanitary conditions. Similarly, the swabbing method may indicate only surface contamination and cannot provide much information on the bacterial cells protected by the membrane structure. In addition, mature biofilm might not be readily detached by such methods. Direct microscopic observations provide evidence of the level of microbial contamination and biofilm formation (Wimpenny et al., 2000; Chmielewski and Frank, 2003). Several microscopy techniques have been used to study biofilm (Wimpenny et al., 2000). Sample preparation for conventional electron microscopy can lead to artifacts, which are revealed in the images. Biofilm contains highly hydrated EPS, which can contain up to $95 \%$ moisture (Serp et al., 2002; Hassan et al., 2003). Severe sample preparation techniques would collapse EPS that are chemically unfixable. However, recent publications still use extensive sample preparations that include fixation in glutaraldehyde, dehydration in alcohol, and critical point drying (Khor et al., 2007). Because of its ability to observe fully hydrated specimen, confocal scanning laser microscopy (CSLM) avoids many sources of artifacts (Hassan et al., 1995). However, the relatively limited resolution of CSLM makes it difficult to obtain information on the detailed structure of biofilm. To our knowledge, no published work has examined the microstructure of biofilm formed on dairy processing membranes. The objective of this study was to evaluate biofilm formation on RO whey concentration membranes by using microscopy techniques that avoided severe sample preparation to minimize artifacts.

\section{MATERIALS AND METHODS}

\section{Membranes}

One each of new, 6-, 12-, and 14-mo-old RO whey concentration membranes were obtained from a whey processing plant. Membranes were collected aseptically after a complete clean in place (CIP) cycle and shipped in polythene bags to the Dairy Science Department, South Dakota State University (Brookings), where they were kept in the cooler $\left(4^{\circ} \mathrm{C}\right)$ until used for microscopy analyses within 3 to $4 \mathrm{~d}$ of arrival. The CIP protocol involved application of alkaline, acid, and enzyme solutions at the end of every 24 -h running cycle.

\section{Membrane Sampling for Microscopic Examinations}

The RO membranes were cut into 3 slices under aseptic conditions using an electric cutter (powered hand saw, Black and Decker, Towson, MD). Each slice was further divided into 3 parts; outer, middle, and inner layers. Although bacterial counts did not differ among the outer, middle, and inner layers (data not shown), the middle layer was used for microscopic examinations to avoid possible environmental contaminants during handling of the membrane. The membrane was cut under sterile conditions into $2 \times 2 \mathrm{~cm}$ pieces for scanning electron microscopy and $1 \times 1 \mathrm{~cm}$ pieces for fluorescence microscopy. Numerous membrane pieces were gently rinsed with phosphate buffer $(\mathrm{pH} 7)$ and observed with the mentioned microscopy techniques.

\section{Fluorescence Microscopy}

Membranes were observed with fluorescence microscopy on the same day they were received. The 14-mo-old membrane pieces were stained with Live/ Dead BacLight Bacterial Viability kit (Invitrogen, Carlsbad, CA). The kit uses a mixture of SYTO 9, a green-fluorescent nucleic acid dye, and propidium iodide, a red-fluorescent nucleic acid dye. This dye mixture differentiates between live and dead bacterial cells based on membrane integrity. Cells with a compromised membrane (dead cells) will stain red, whereas cells with an intact membrane will stain green. The excitation and emission maxima for these dyes are about 480 and $500 \mathrm{~nm}$, respectively, for SYTO 9 and 490 and $635 \mathrm{~nm}$, respectively, for propidium iodide. Dyes were prepared according to the manufacturer's instructions (1 applicator pipette from each dye was added to $5 \mathrm{~mL}$ of filter-sterilized deionized water). However, based on preliminary experiments, the optimum final concentration of the dyes was one-half that recommended by the manufacturer. Biofilm formed on membrane was also stained by a wheat germ agglutinin fluorescent conjugate (Invitrogen), one of the most successful lectins used to label bacterial EPS (Hassan et al., 2002). The excitation and emission maxima for this dye are 650 and $665 \mathrm{~nm}$, respectively. A stock solution was prepared by dissolving $1 \mathrm{mg}$ of the dye in $1 \mathrm{~mL}$ of $0.1 \mathrm{M}$ sodium bicarbonate solution. The working solution was prepared by diluting the stock solution 10 times in 0.1 $M$ sodium bicarbonate solution. Fluorescent dyes were applied to the membrane pieces, which were then incubated in the dark for $30 \mathrm{~min}$, followed by rinsing with phosphate buffer 3 times and observed with a Leica fluorescence microscopy (North Central Instruments Inc., Minneapolis, MN). Images were captured with a 

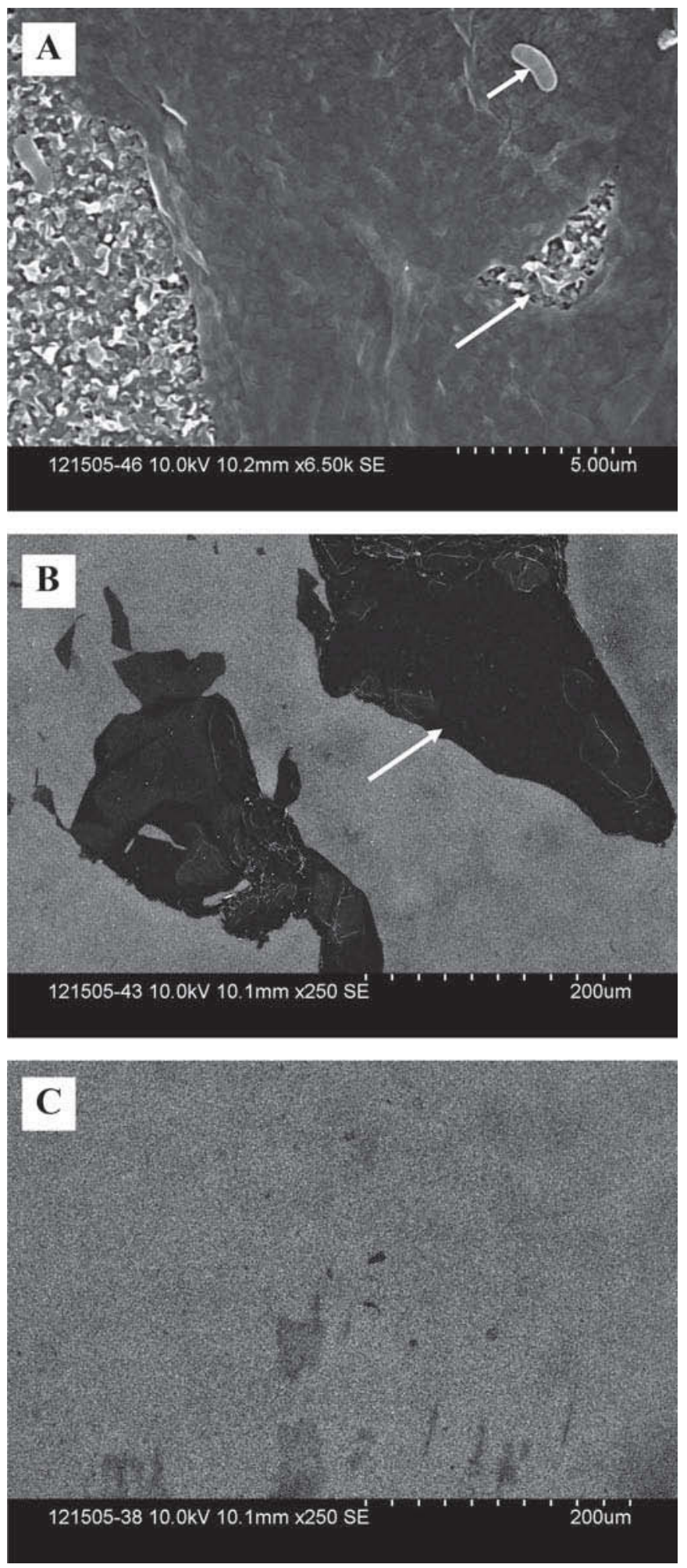

Figure 1. Scanning electron micrographs of unused reverse osmosis whey concentration membrane showing bacterial cells attached to the surface (A), differences in structure between the polyamide barrier and the hydrophilic coating (B), and whole microscopic field showing minimum coating $(\mathrm{C})$. In figure $\mathrm{A}$, short arrow points to a bacterial cell and long arrow points to area not covered by the hydrophilic coating. In figure B, arrow points to the hydrophilic coating.
QImaging digital camera (Texas Instruments, Dallas, TX) and analyzed by Image Pro software (version 4.5, Media Cybernetics Inc., Bethesda, MD).

\section{Scanning Electron Microscopy}

To minimize the structural damage to biofilm, a modified air drying method was used to obtain a partially dehydrated biofilm for electron microscopy. After gentle rinsing with phosphate buffer ( $\mathrm{pH} 7.0$ ), numerous (3-4) membrane pieces were air dried at $22^{\circ} \mathrm{C}$ under laminar air flow for $24 \mathrm{~h}$. Samples were sputter coated with a 5-nm layer of gold using a CRC-150 sputtering system (Plasma Science Inc., Lorton, VA). Unused new membrane pieces were directly coated with gold without the drying step. Specimens were observed with a Hitachi scanning electron microscope (model S-3400N, Hitachi SCI Systems Ltd., Tokyo, Japan) at 5 to $10 \mathrm{kV}$.

\section{RESULTS AND DISCUSSION}

Figure 1 shows the microstructure of the unused polyamide RO membrane used for whey processing. The image in Figure 1A indicates that the new membrane itself could serve as a source of bacteria. The only treatment applied to new membranes in some whey processing plants is warm water rinsing. This would not be sufficient to remove cells attached to the membrane surface. The initial contaminants of the membrane, along with bacterial cells that survived pasteurization in the feed, could thus serve as the source of biofilms in dairy separation systems.

Surface modifications to reduce free energy, adhesion, and stickiness are now considered the most ecologyfriendly approaches to control fouling (Vladkova, 2007). One of the most common examples is a hydrophilic surface that reduces protein absorption because of lack of hydrophobic interactions (Gölander et al., 1986). According to the membrane manufacturer, a less coarse hydrophilic layer coats the polyamide barrier to prevent plugging and changes the surface properties of the membrane with the aim of reducing hydrophobic interactions and fouling. Our microscopic observations showed that less than $50 \%$ of the surface was covered by this hydrophilic layer, as indicated by dark areas on the new membrane (Figures $1 \mathrm{~B}$ and $\mathrm{C}$ ). An interesting observation was that some deposits (proteins or mineral-proteins) were seen only on the polyamide areas, not covered by the hydrophilic sheet (Figure 2). This indicates that the manufacturer approach of forming a hydrophilic layer on the surface of the membrane is successful in reducing interactions between deposits (proteins) and the membrane. However, the image in Figure 3 shows that single cells or cells in short chains 


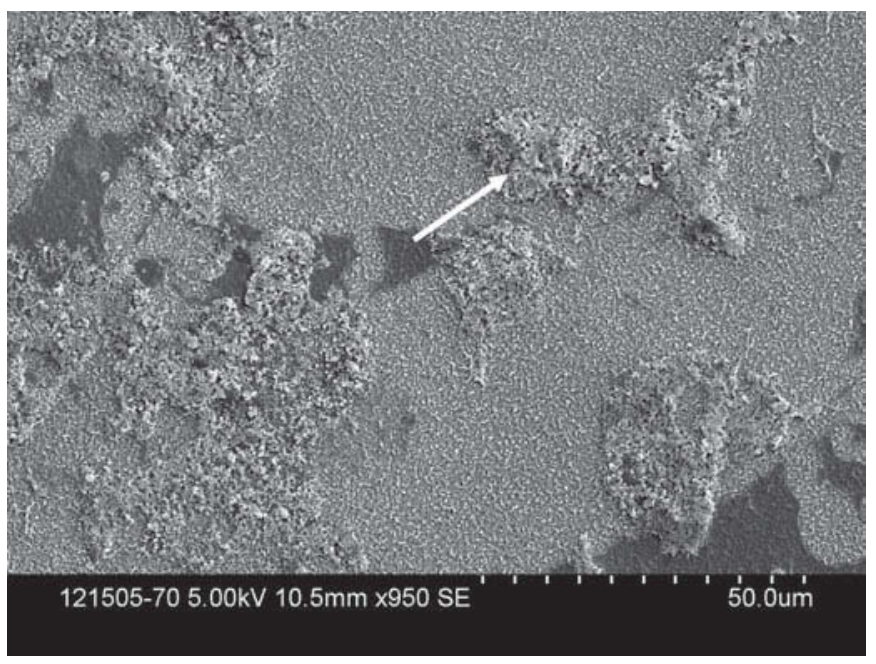

Figure 2. Foulants absorbed on the surface of the polyamide barrier on the 12-mo-old membrane as observed by scanning electron microscopy. Arrow points to deposits that seem to be protein aggregates.

attached mainly to the hydrophilic areas. Nonetheless, microscopic observations could not provide evidence that biofilm formed only on the hydrophilic layer. It is possible that the initial attachment takes place on the hydrophilic layer. It is generally accepted that hydrophilic surfaces support bacterial attachment and biofilm formation more than hydrophobic surfaces (Blackman and Frank, 1996; Sinde and Carballo, 2000; Chmielewski and Frank, 2003). However, contrary conclusions have also been reported (Pasmore et al., 2001). Bos et al. (2000) reported that although hydrophobicity did not affect bacterial adhesion, it was a major retention de-

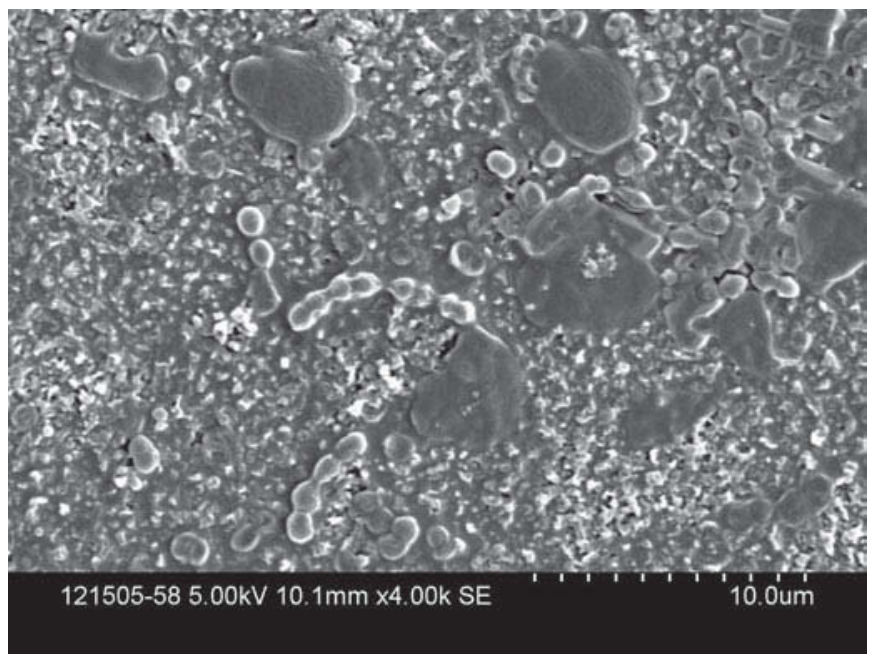

Figure 3. Scanning electron micrographs showing bacterial cells attached to the coating on the 12-mo-old reverse osmosis whey concentration membrane. terminant. All commercially available release coatings decreased adhesion of foulants, but none could prevent biofilm formation (Vladkova, 2007). The ability of a bacterial cell to attach may also depend on the cell surface properties, such as charge, hydrophobicity, and capsule formation (Hassan and Frank, 2004).

Three distinct multispecies biofilm structures were observed on the RO whey processing membranes (Figure 4): 1) a monolayer of rods and cocci of different sizes connected by extracellular polymers (Figure 4A), 2) a 3-dimensional structure of a dense matrix of EPS where different types of bacterial cells were embedded (Figures $4 \mathrm{~B}$ and $\mathrm{C}$ ), and 3) thick aggregates of rough surface bacterial cells with no visible EPS matrix (Figure 4D). The uneven surface of the bacterial cells in Figure 4D and bridges between cells might indicate the presence of EPS, which could act as a cementing agent and hold cells together. The reason bacteria form different structures of biofilm is not fully understood. The differences in microbial species forming biofilm and the type of polymer they secrete are important factors affecting the nature and structure of biofilm (Chmielewski and Frank, 2003). Also, bacteria tend to form a monolayer biofilm on hydrophilic surfaces (McEldowney and Fletcher, 1987). In addition, nutrient and redox potential gradients would be expected in thick biofilm. Therefore, anaerobic and slow-growing bacteria might be found in deep layers of thick aggregates of biofilm (Wimpenny et al., 2000; Cemielewski and Frank, 2003). Generally, multispecies biofilm is thicker and more stable, which might be because of production of a variety of EPS from the different bacterial species. The differences in the structure of biofilm in Figure 4 could be related to variations in the amount or type of EPS. In Figures 4B and C, a well-defined matrix of EPS, which is partially dehydrated in some areas because of sample preparation, is apparent. However, the EPS in biofilm shown in Figure 4D might be produced in a limited amount, or EPS could be of a different structure from those in Figures 4B and C. Biofilms were observed to be unevenly distributed on the surface of the membrane. This may possibly be attributed to the differences in the surface properties of the membrane from one area to another.

Under appropriate growth conditions, bacteria may develop organized structures (maturation). Different models have been proposed for mature biofilm structure. A mushroom-shaped structure was suggested based on CSLM observations of water systems (Wimpenny et al., 2000). Figure 5 shows a mushroom-shaped structure of biofilm formed on RO whey concentration membranes. The image in Figure $5 \mathrm{~B}$ shows that the structure of biofilm formed on 6-mo-old membranes can be best described as hills and valleys, with hills 
having mushroom-like structures. The valleys comprised bacterial cells with different shapes embedded in a thick matrix of EPS. In the mushroom structure, a shell covered cell aggregates, possibly because of excessive secretion of EPS (Figure 5A). Figure 5C shows 2 different types of structure on the 14-mo-old membrane. A very dense cell aggregate was formed on a matrix of multilayer, mixed-species biofilm. This study is the first to observe the above structures on dairy membranes. Furthermore, no reports showed formation of more than 1 structure of biofilm on 1 surface. Our study clearly demonstrates that formation of different biofilm structures on 1 membrane would be possible. It was interesting to note that the polymeric materials extended from the cell aggregate to an individual chain of cocci (Figure 5C). A cell could have detached from the aggregates and formed the chain, or another possibility is that the cell aggregate recruited the chain to become part of the biofilm. Figure 5D shows that part of the shell layer broke off and the cells detached. Partial dehydration during sample preparation might lead to structure shrinkage but is unlikely to lead to the detachment of biofilm parts. It is hypothesized that detached cells may repopulate another area and form a new biofilm. Bacteria are known to detach from biofilm through the process of sloughing and shearing. After reaching a certain thickness, accumulation of gases and acids, in addition to the effect of shearing, causes sloughing of the polymer layer responsible for biofilm stability (Bryers, 1987; Chmielewski and Frank, 2003).
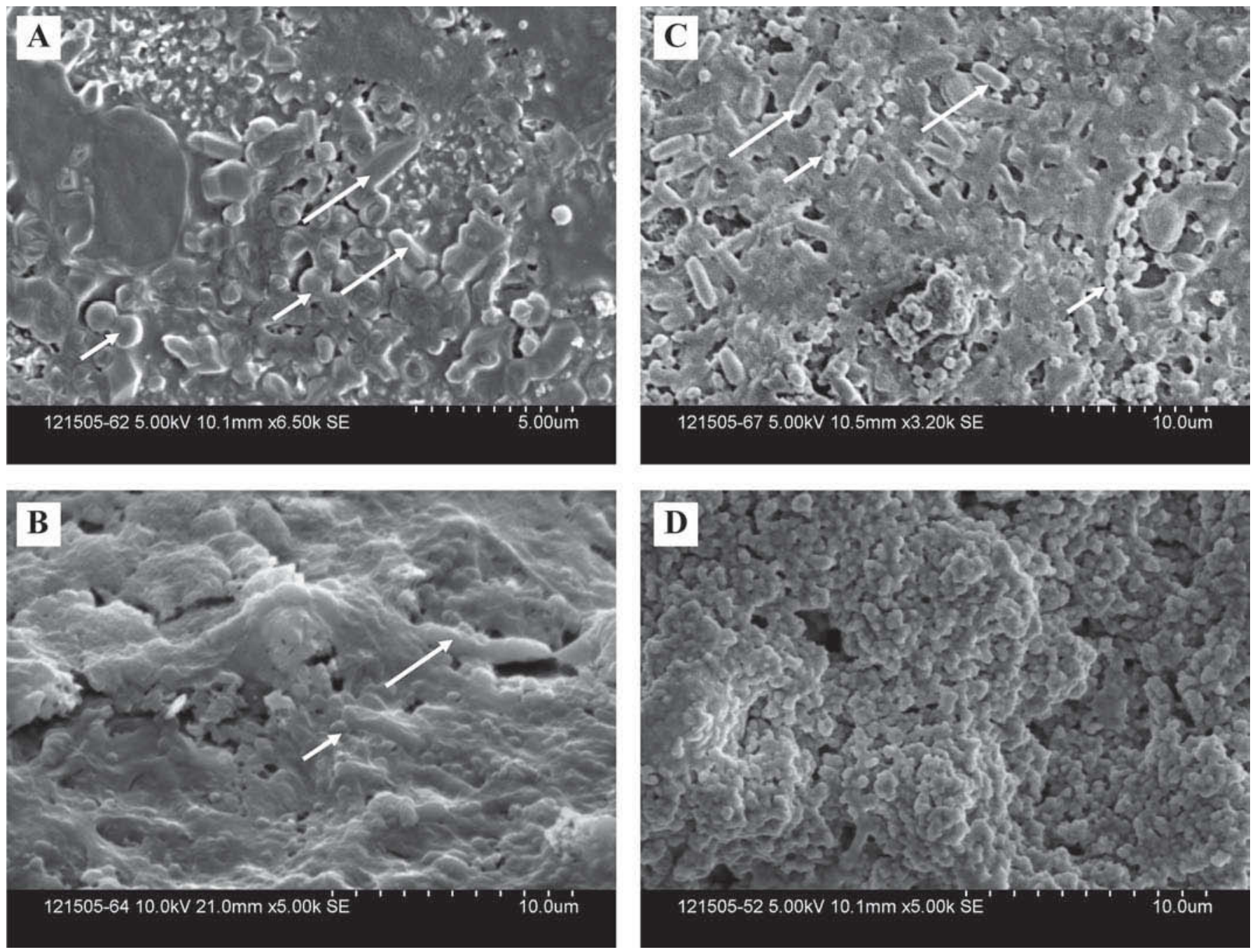

Figure 4. Scanning electron micrographs of biofilm formed on 6-, 12-, and 14-mo-old reverse osmosis whey concentration membranes, Monolayer of rods and cocci of different sizes connected by extracellular polymers on a 12-mo-old membrane (A), a 3-dimensional structure of a dense matrix of extracellular polymeric substances where different types of bacterial cells were embedded on a 6-mo-old membrane (B), layers of a mixed culture of rods and cocci biofilm formed on a 14-mo-old membrane $(\mathrm{C})$, and thick aggregates of rough surface bacterial cells with no visible extracellular polymeric substance matrix on 12-mo-old membrane (D). Long arrows point to rods and small arrows point to cocci. 
In addition, low nutrient availability can increase EPS production to levels that could lead to detachment (Kim and Frank 1995; Chmielewski and Frank, 2003).

We observed biofilm on membranes after completion of a CIP cycle, which involved application of disinfectants. Scanning electron microcopy observations could not differentiate between live cells and dead cells. Also, because mature biofilm might not be easy to remove, plate counting following swabbing might not provide reliable information on the level of bacterial survival after cleaning and sanitation. Therefore, fluorescence microscopy combined with live/dead bacterial stain was used to observe viable cells in biofilm. Figure 6 shows biofilm formed on a 14-mo-old membrane strained with the live/dead dye. Live bacterial cells were seen on all membranes tested in this study (6-, 12-, and 14-mo). Live and dead cells did not seem to be segregated, which might indicate the absence of a protective role of membrane structures and differences in resistance to disinfectants. Considering that the fluorescence microscopy allows observation of cells only on the top layer of the biofilm, it was interesting to see live cells even on the surface, which was in direct contact with disinfectants during the CIP cycle. This indicates that biofilm becomes recalcitrant to disinfectants and extremely difficult to eradicate and can serve as a reservoir of detached cells that would contaminate the concentrated product.
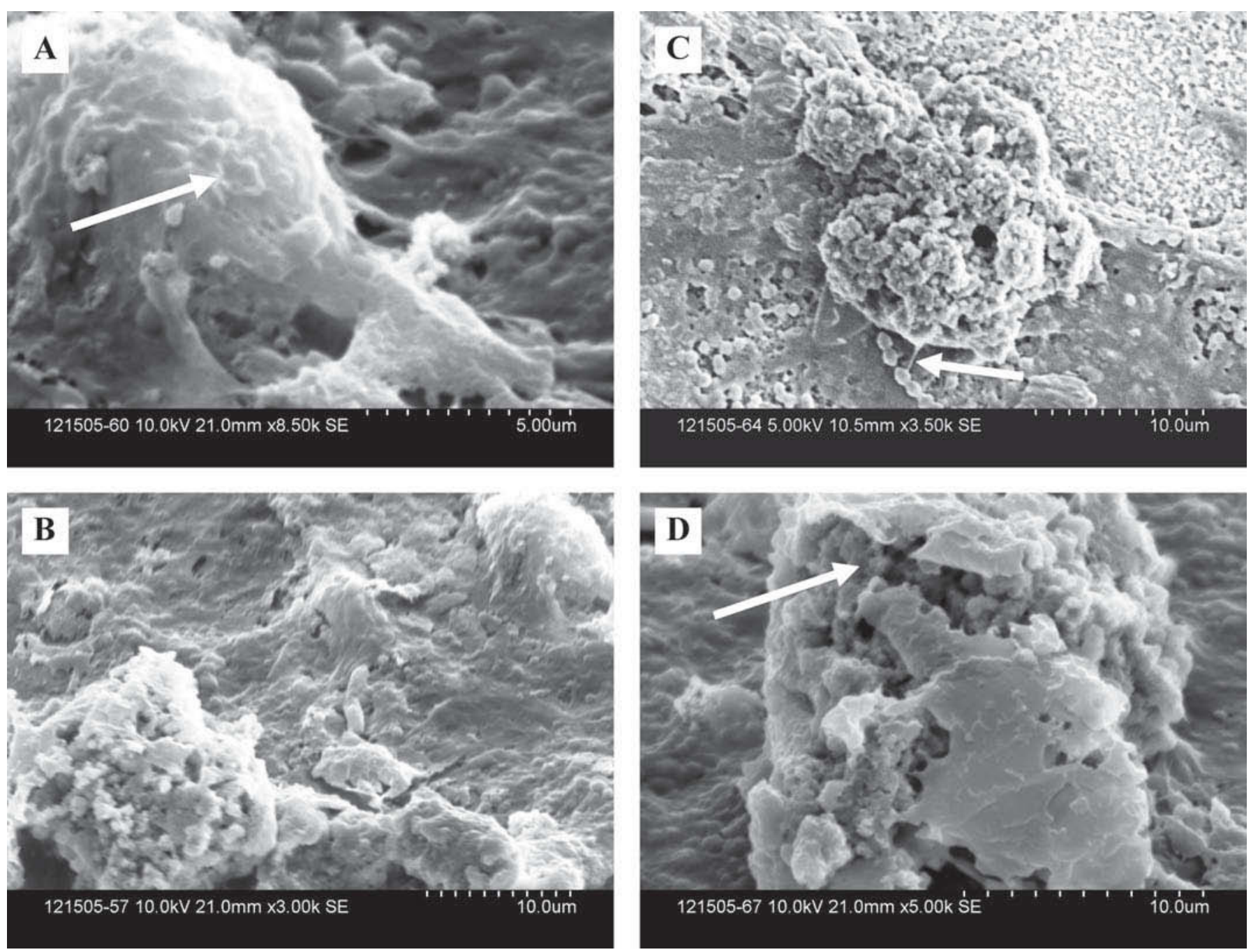

Figure 5. A mushroom-like structure of mature biofilm formed on 6- and 14-mo-old reverse osmosis whey concentration membrane. Wrinkled shell covering cell aggregates in a 6-mo-old membrane (A), a hill and valley structure of biofilm on a 6-mo-old membrane (B), a biofilm on a 14-mo-old membrane showing 2 types of biofilm structures, large aggregates and a dense matrix of microorganisms (C), and a part of the mature biofilm was broken off, indicating detachment of some bacterial cells to repopulate another area and possibly form a new biofilm (D). Arrow in A points to wrinkled shell, in $\mathrm{C}$ points to polymeric materials extended from the aggregated cells to a chain of cocci, and in D points to a broken area in the mature biofilm. 


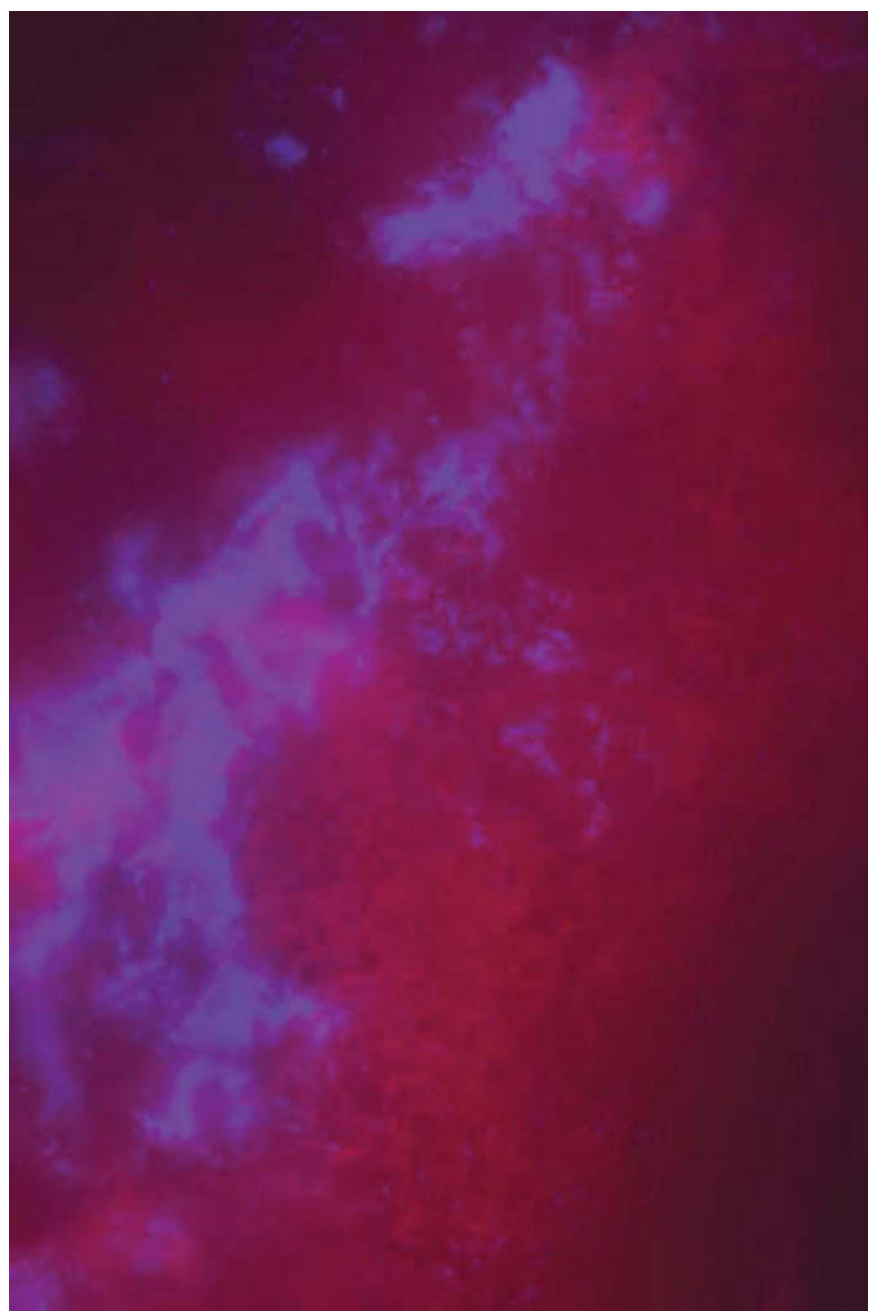

Figure 6. Fluorescence microscopy image showing dead (black; red in color version) and live (gray; blue in color version) bacterial cells on the surface of 14-mo-old reverse osmosis whey concentration membrane. Color version available in online PDF.

Wheat germ agglutinin conjugated with a fluorescent dye was successfully used to observe biofilm on the RO membrane (Figure 7). The dye labeled a structure on the surface of bacterial cells (Figure 7A), which could be capsules. It also stained whole areas covered by biofilm (Figure 7B). Long chains of rods (more than 50 cells) were observed by both fluorescence (Figure 8A) and scanning electron microscopy (Figure 8B and C). Formation of long chains in biofilm has previously been reported (Dalton et al., 1994; Vejborg and Klemm, 2009). A marine bacterium formed a biofilm consisting of single and paired cells on hydrophobic surfaces, but it formed chains up to 100 um on hydrophilic substratum (Dalton et al., 1994). However, chain formation by Escherichia coli was not affected by the properties of the attachment site surface but rather was the result of intercellular interactions mediated by an autotrans- porter protein implicated in biofilm formation (Vejborg and Klemm, 2009). In our study, long chains seemed to be found on the relatively more hydrophilic areas of the membrane. The formation of long chains might increase coherence and resistance of cells under conditions such as high shearing (Vejborg and Klemm, 2009). In addition to formation of long chains, bacterial cells organized in a very unusual pattern on the 14-mo-old membrane (Figure 8D). X-ray analysis indicated that the structure in Figure 8D was not mineral deposits. It seems that, in addition to formation of long chains, bacterial cells organized in such a unique pattern for unknown reason. Partial dehydration because of sample preparation might have contributed, in part, to the formation of such structures.
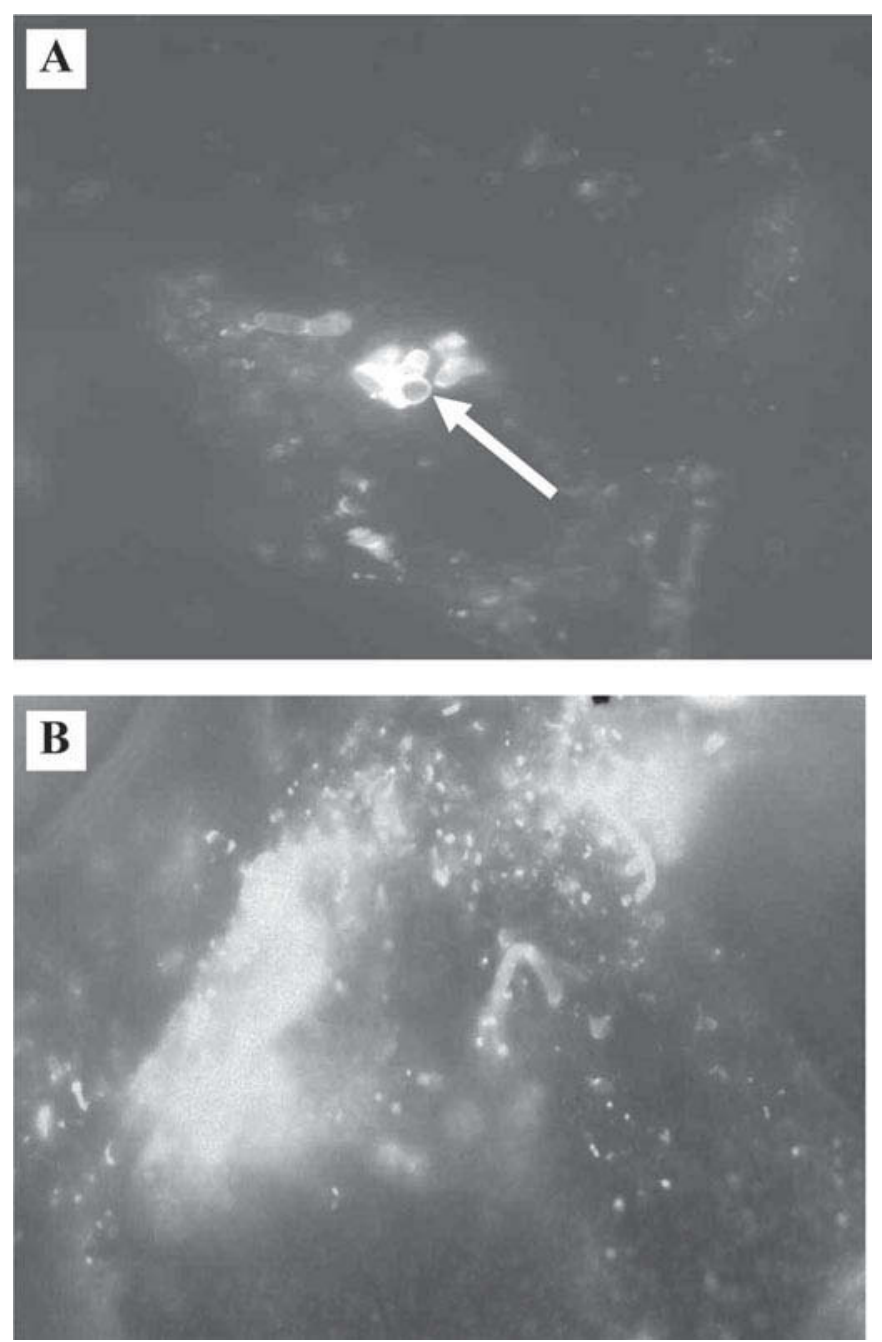

Figure 7. Fluorescence microscopy images of biofilm on a 6-mo-old membrane stained with a wheat germ agglutinin fluorescent conjugate. The surface of bacterial cells labeled with the dye (A), and the whole biofilm stained with the dye (B). Arrow points to cell surface labeled with the wheat germ agglutinin. 

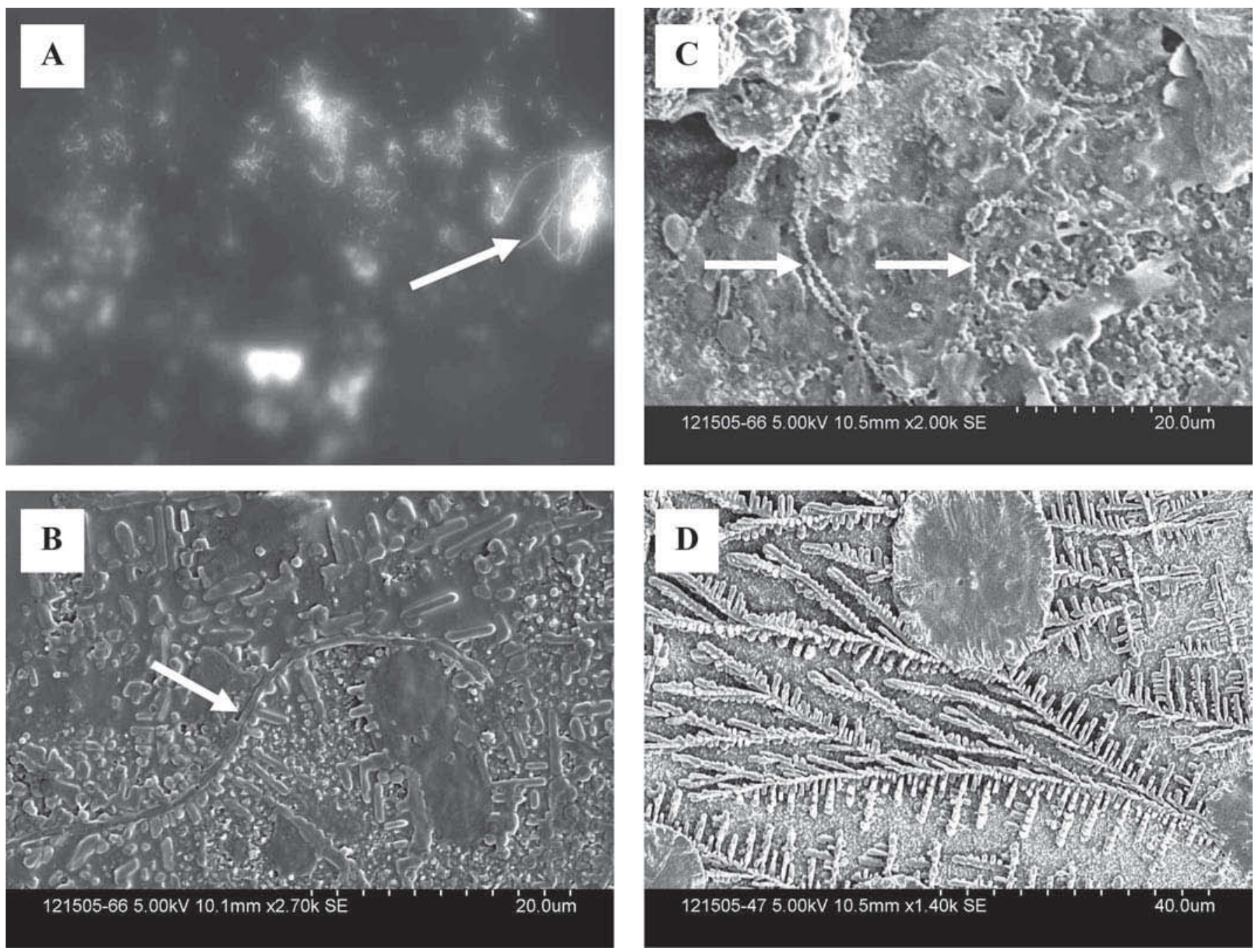

Figure 8. Long chains of bacteria cells attached to the reverse osmosis whey concentration membranes as observed by fluorescence microscopy and scanning electron microscopy. Fluorescence image of a 12-mo-old membrane (A), scanning electron microscopy image of a 12-mo-old membrane (B), and scanning electron microscopy images of a 14-mo-old membrane (C and D). Arrows point to bacterial chains.

\section{CONCLUSIONS}

The study directly observes, for the first time, structure of biofilm formed in vivo on RO whey concentration membranes. Because biofilm principles derived from nonfood systems may not apply to a nutrient-rich food environment, the information obtained from the current study would be useful for the dairy industry in developing more effective cleaning and sanitation regimens customized to dairy systems. The extent of biofilm formation on RO whey concentration membranes and the level of viable cells, as observed by microscopy, could be a result of high counts of microorganisms surviving pasteurization or of postpasteurization growth and ineffective CIP protocols. The 3 phenotypes observed with scanning electron microscopy suggest that different types of microorganisms and their EPS were involved in biofilm formation. We are currently studying the relationship between biofilm structure and its dislodging and resistance to disinfectants.

\section{ACKNOWLEDGMENTS}

The authors thank Dorin Cengher (Electrical Engineering and Computer Science Department, South Dakota State University, Brookings) for help with the scanning electron microscope.

\section{REFERENCES}

Blackman, I. C., and J. Frank. 1996. Growth of Listeria monocytogenes as a biofilm on various food processing surfaces. J. Food Prot. 59:827-839.

Bos, R., H. C. van der Mei, J. Gold, and H. J. Busscher. 2000. Retention of bacteria on a substratum surface with micro-patterned hydrophobicity. FEMS Microbiol. Lett. 189:311-315. 
Bryers, J. D. 1987. Biologically active surfaces: Processes governing the formation and persistence of biofilms. Biotechnol. Prog. 3:5768.

Chmielewski, R. A. N., and J. F. Frank. 2003. Biofilm formation and control in food processing facilities. Comprehensive Rev. Food Sci. Food Safety 2:22-32.

Dalton, H. M., L. K. Poulsen, P. Halasz, M. L. Angles, A. E. Goodman, and K. C. Marshall. 1994. Substratum-induced morphological changes in a marine bacterium and their relevance to biofilm structure. J. Bacteriol. 176:6900-6906.

Gölander, C., S. Jönsson, T. Vladkova, P. Stenius, and J. C. Eriksson. 1986. Preparation and protein adsorption properties of photopolymerized hydrophilic films containing $N$-vinylpyrrolidone (NVP), acrylic acid (AA) or ethyleneoxide (EO) units as studied by ESCA. Colloids Surf. 21:149-165.

Hassan, A. N., J. F. Frank, and K. B. Qvist. 2002. Direct observation of bacterial exopolysaccharides in dairy products using confocal scanning laser microscopy. J. Dairy Sci. 85:1705-1708.

Hassan, A. N., and J. F. Frank. 2004. Attachment of Escherichia coli O157:H7 grown in tryptic soy broth and nutrient broth to apple and lettuce as related to cell hydrophobicity, surface charge and capsule production. Int. J. Food Microbiol. 96:103-109.

Hassan, A. N., J. F. Frank, and M. Elsoda. 2003. Observation of bacterial exopolysaccharides in dairy products using cryo-scanning electron microscopy. Int. Dairy J. 13:755-762.

Hassan, A. N., J. F. Frank, M. A. Farmer, K. A. Schmidt, and S. I. Shalabi. 1995. Formation of yogurt microstructure and three dimensional visualization as determined by confocal scanning laser microscopy. J. Dairy Sci. 78:2629-2636.

Herzberg, M., and M. Elimelech. 2007. Biofouling of reverse osmosis membranes: Role of biofilm-enhanced osmotic pressure. J. Membr. Sci. 295:11-20.

Khor, S. L., D. D. Sun, Y. Liu, and J. O. Leckie. 2007. Biofouling development and rejection enhancement in long SRT MF membrane bioreactor. Process Biochem. 42:1641-1648.

Kim, K. Y., and J. F. Frank. 1995. Effect of nutrients on biofilm formation by Listeria monocytogenes on stainless steel. J. Food Prot. 58:24-28.
McEldowney, S., and M. Fletcher. 1987. Adhesion of bacteria from mixed cell suspension to solid surfaces. Arch. Microbiol. 148:5762.

Muthukumaran, S., S. Kentish, S. Lalchandani, M. Ashokkumar, R. Mawson, G. W. Stevens, and F. Grieser. 2005. The optimisation of ultrasonic cleaning procedures for dairy fouled ultrafiltration membranes. Ultrason. Sonochem. 12:29-35.

Pasmore, M., P. Todd, S. Smith, D. Baker, J. Silverstein, D. Coons, and C. N. Bowman. 2001. Effects of ultrafiltration membrane surface properties on Pseudomonas aeruginosa biofilm initiation for the purpose of reducing biofouling. J. Membr. Sci. 194:15-21.

Ridgway, H. F., A. Kelly, C. Justice, and B. H. Olson. 1983. Microbial fouling of reverse-osmosis membranes used in advanced wastewater treatment technology: Chemical, navteriological, and ultrastructural analyses. Appl. Environ. Microbiol. 45:1066-1084.

Serp, D., M. Mueller, U. von Stockar, and I. W. Marison. 2002. Low temperature electron microscopy for the study of polysaccharideultrastructures in hydrogels. I. Theoretical and technical considerations. Biotechnol. Bioeng. 79:243-252.

Sinde, E., and J. Carballo. 2000. Attachment of Salmonella spp. and Listeria monocytogenes to stainless steel, rubber and polytetrafluorethylene: The influence of free energy and the effect of commercial sanitizers. Food Microbiol. 17:439-447.

Stewart, P. S., and J. W. Costerton. 2001. Antibiotic resistance of bacteria in biofilms. Lancet 358:135-138.

Tang, X., S. H. Flint, J. D. Brooks, and R. J. Bennett. 2009. Factors affecting the attachment of micro-organisms isolated from ultrafiltration and reverse osmosis membranes in dairy processing plants. J. Appl. Microbiol. 107:443-451.

Vejborg, R. M., and P. Klemm. 2009. Cellular chain formation in Escherichia coli biofilms. Microbiology 155:1407-1417.

Vladkova, T. 2007. Surface engineering for non-toxic biofouling control. J. U. Chem. Technol. Metallurgy 42:239-256.

Wimpenny, J., W. Manz, and U. Szewzyk. 2000. Heterogeneity in biofilms. FEMS Microbiol. Rev. 24:661-671. 